\title{
IDENTITIES IN COMBINATORICS. II: A $q$-ANALOG OF THE LAGRANGE INVERSION THEOREM
}

\author{
GEORGE E. ANDREWS ${ }^{1}$
}

ABSTRACT. A q-analog of Lagrange's inversion theorem is obtained. It is applied to give a new proof of an expansion theorem due to Carlitz and to obtain formulae for certain combinatorial numbers studied by Carlitz.

1. Introduction. In [3], T. Carlitz addressed the question of possible $q$-analogs for the celebrated Lagrange inversion formula [1, p. 158]:

$$
f(x)=f(0)+\sum_{n=1}^{\infty} \frac{x^{n}}{n !(\phi(x))^{n}}\left[\frac{d^{n-1}}{d t^{n-1}}\left(f^{\prime}(t)(\phi(t))^{n}\right)\right]_{t=0},
$$

a formula valid provided $f(x)$ and $\phi(x)$ are analytic around $x=0$ and $\phi(0) \neq 0$.

Carlitz [3, p. 206, equation (1.11)] was able to show that

$$
f(x)=f(0)+\sum_{n=1}^{\infty} \frac{x^{n}}{(q)_{n}(x)_{n}}\left[\Delta^{n-1}\left(\Delta f(t) \cdot(t)_{n}\right)\right]_{t=0},
$$

where $(x)_{n}=(1-x)(1-x q) \cdots\left(1-x q^{n-1}\right)$ and $\Delta f(t)=t^{-1}(f(t)-f(t q))$. This result is a $q$-analog of $(1.1)$ in the case $\phi(x)=1-x$.

In [4], Carlitz asks for a general $q$-analog of Iagrange inversion. Namely he asks for a formula for the $C_{n}$ in

$$
F(x)=\sum_{n \geq 0} \frac{C_{n} x^{n}}{(q)_{n} \phi(x q) \phi\left(x q^{2}\right) \cdots \phi\left(x q^{n}\right)}, \quad C_{0}=F(0) .
$$

In particular he asks for an operational formula for computing the $C(n \mid q)$ in

$$
x=\sum_{n=1}^{\infty} x^{n}(x q){ }_{n} C(n-1 \mid q)
$$

and he points out that

$$
C(n-1 \mid q) \neq \frac{(1-q)}{(q)_{n}}\left\{\Delta^{n-1}(x q)_{n}^{-1}\right\}=q^{n-1} \frac{(1-q)}{\left(1-q^{n}\right)}\left(\begin{array}{c}
2 n-2 \\
n-1
\end{array}\right)_{q},
$$

where $\left(\begin{array}{l}N \\ M\end{array}\right)_{q}=(q)_{N}(q)_{M}^{-1}(q)_{N-M}^{-1}$.

In [2], Carlitz discusses $C(n \mid q)$ extensively, which is initially defined by

$$
C(n \mid q)=\sum_{\left(a_{i}\right)} q^{a_{1}+a_{2}+\cdots+a_{n}}
$$

Received by the editors September 3, 1974 and, in revised form, October 16, 1974. AMS (MOS) subject classifications (1970). Primary 05A10, 05A15, 05A19, 33A30. Key words and phrases. Lagrange inversion formula, $q$-analogs, Catalan numbers. ${ }^{1}$ Partially supported by NSF Grant GP-23774. 
summed over all $\left(a_{1}, a_{2}, \cdots a_{n}\right)$ with $1 \leq a_{1} \leq a_{2} \leq \cdots \leq a_{n}$ and $a_{i} \leq i$ for each $i$.

When $q=1$, then, of course, the Lagrange inversion formula (1.1) is applicable to (1.3) and indeed $C(n \mid 1)=(n+1)^{-1}\left(\begin{array}{c}2 n \\ n\end{array}\right)$, the $n$th Catalan number.

In this paper we shall (in $\$ 2$ ) prove the following $q$-analog of the Lagrange inversion formula (1.1); our result reduces to (1.2) in the case $\phi(x)=1-x q^{-1}$ and it reduces to (1.1) when $q \rightarrow 1$. Before stating our main result we require the following notation:

$$
\Delta_{(n)} f(t)=\left(f(t)-f\left(t q^{n}\right)\right) / t
$$

Note that $\Delta=\Delta_{(1)}$; indeed $\Delta_{(n)}$ is just $q$-differentiation to the base $q^{n}$. Furthermore $\Delta_{(0)} f(x) \equiv 0$.

Theorem 1. Let $F(x)$ and $\phi(x)$ be analytic around $x=0$ with $\phi(0) \neq 0$, and let $k$ be a nonnegative integer. Define

$$
\begin{aligned}
\delta_{r} & =(q)_{r-1} \cdot\left\{\text { Coefficient of } t^{r-1} \text { in }(\Delta F(t)) \cdot \phi\left(t q^{k}\right) \phi\left(t q^{k+1}\right) \cdots \phi\left(t q^{k+r-1}\right)\right\} \\
& =\left[\Delta^{r-1}\left((\Delta F(t)) \phi\left(t q^{k}\right) \phi\left(t q^{k+1}\right) \cdots \phi\left(t q^{k+r-1}\right)\right)\right]_{t=0},
\end{aligned}
$$

and for $j \leq r$,

$$
\begin{aligned}
\epsilon_{j}(r)= & -(q)_{r-1} q^{r-j} \\
& \cdot\left\{\text { Coefficient of } t^{j-2} \text { in }\left(\Delta_{(r-j)} t^{-1} \phi(t q)\right) \frac{\phi\left(t q^{k}\right) \phi\left(t q^{k+1}\right) \cdots \phi\left(t q^{k+r-1}\right)}{\phi(t q) \phi\left(t q^{2}\right) \cdots \phi\left(t q^{r-j+1}\right)(q)_{r-j}}\right\} \\
= & -\left(q^{j-1}\right)_{r-j+1} q^{r-j} \\
& \cdot\left[\Delta^{j-2}\left\{\left(\Delta_{(r-j)}{ }^{-1} \phi(t q)\right) \frac{\phi\left(t q^{k}\right) \phi\left(t q^{k+1}\right) \cdots \phi\left(t q^{k+r-1}\right)}{\phi(t q)^{\prime}\left(t q^{2}\right) \cdots \phi\left(t q^{r-j+1}\right)(q)_{r-j}}\right\}\right]_{t=0} \cdot
\end{aligned}
$$

Then, for $|q|<1$,

$$
F(x)=F(0)+\sum_{n=1}^{\infty} \frac{x^{n} C_{n}}{(q)_{n} \phi(x q) \phi\left(x q^{2}\right) \cdots \phi\left(x q^{n}\right)},
$$

where

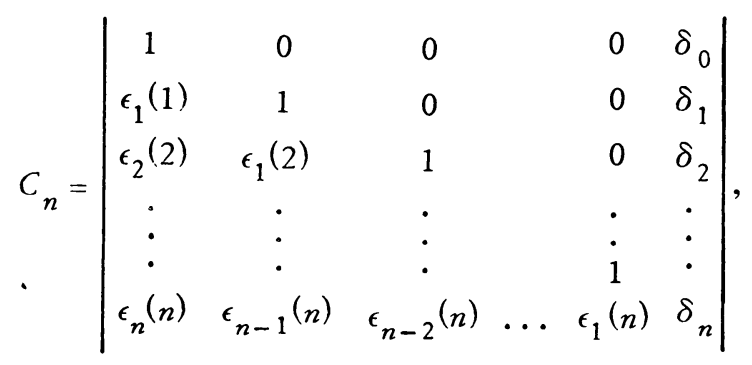

When $q \rightarrow 1$, we shall show that $\epsilon_{j}(n)(q)_{j}^{-1} \rightarrow 0$ whenever $j<n$; hence it follows that 


$$
C_{n}(q)_{n}^{-1} \rightarrow n !^{-1}\left[\left(d^{n-1} / d t^{n-1}\right)\left(f^{\prime}(t)(\phi(t))^{n}\right)\right]_{t=0}
$$

as $q \rightarrow 1$ (see (2.4) at the end of $\$ 2$ ). Thus Theorem 1 is the $q$-analog of Lagrange inversion.

In $\$ 3$, we shall apply Theorem 1 to prove $(1.2)$ and certain formulae related to Carlitz's work in [3]. In $\$ 4$, we shall apply Theorem 1 to the $C(n \mid q)$.

2. Proof of Theorem 1. The elementary proof of (1.1) found in [1, \$55.1] will be adapted to our needs here. We begin with the observation that the $C_{n}$ in (1.4) must exist since $\left|x^{n} / \phi(x q) \cdots \phi\left(x q^{n}\right)\right|<\rho^{n}<1$ for $x$ in some sufficiently small neighborhood of 0 . Replacing $x$ by $t$ in (1.4) and applying $\Lambda$ we see that

$$
\begin{aligned}
\Delta F(t) & =\sum_{n \geq 0} \frac{C_{n} t^{n-1}}{(q)_{n}}\left\{\frac{1}{\phi(t q) \phi\left(t q^{2}\right) \cdots \phi\left(t q^{n}\right)}-\frac{q^{n}}{\phi\left(t q^{2}\right) \phi\left(t q^{3}\right) \cdots \phi\left(t q^{n+1}\right)}\right\} \\
& =\sum_{n \geq 0} \frac{C_{n} t^{n} q^{n}\left\{t^{-1} q^{-n} \phi\left(t q^{n+1}\right)-t^{-1} \phi(t q)\right\}}{(q)_{n} \phi(t q) \phi\left(t q^{2}\right) \cdots \phi\left(t q^{n+1}\right)} \\
& =-\sum_{n \geq 0} \frac{C_{n} t^{n+1} q^{n} \Delta_{(n)}\left[t^{-1} \phi(t q)\right]}{(q)_{n} \phi(t q) \phi\left(t q^{2}\right) \cdots \phi\left(t q^{n+1}\right)} .
\end{aligned}
$$

Hence

$$
\begin{aligned}
& (\Delta F(t)) \phi\left(t q^{k}\right) \phi\left(t q^{k+1}\right) \cdots \phi\left(t q^{k+r-1}\right)_{t}^{-r}(q)_{r-1} \\
& \quad=-(q)_{r-1} \sum_{n \geq 0} \frac{C_{n} t^{n-r+1} q^{n}\left(\Delta_{(n)} t^{-1} \phi(t q)\right) \phi\left(t q^{k}\right) \phi\left(t q^{k+1}\right) \cdots \phi\left(t q^{k+r-1}\right)}{(q)_{n} \phi(t q) \phi\left(t q^{2}\right) \cdots \phi\left(t q^{n+1}\right)}
\end{aligned}
$$

We now ask for the coefficient of $t^{-1}$ on each side of the above identity. On the left-hand side we see that $\delta_{r}$ is the coefficient of $t^{-1}$. On the right-hand side we see that for $n>r$ each term is analytic in $t$ and so the coefficient of $t^{-1}$ is zero; hence we need only examine those terms with $n \leq r$. When $n=r$, the term is

$$
\frac{C_{r} t^{-1}\left\{\phi^{\prime}\left(t q^{r+1}\right)-q^{r} \phi(t q)\right\} \phi\left(t q^{k}\right) \cdots \phi\left(t q^{k+r-1}\right)}{\left(1-q^{r}\right) \phi(t q) \phi\left(t q^{2}\right) \cdots \phi\left(t q^{r+1}\right)}=\frac{C_{r} t^{-1}}{1-q^{r}}\left(1-q^{r}+\alpha t+\beta t^{2}+\cdots\right)
$$

and so the coefficient here is $C_{r}$.

When $n<r$, we desire the coefficient of $t^{r-n-2}$ in

$$
-(q)_{r-1} C_{n} q^{n}\left(\Delta_{(n)} t^{-1} \phi(t q)\right) \frac{\phi\left(t q^{k}\right) \phi\left(t q^{k+1}\right) \cdots \phi\left(t q^{k+r-1}\right)}{\phi(t q) \phi\left(t q^{2}\right) \cdots \phi\left(t q^{n+1}\right)(q)_{n}}
$$

which is just $C_{n} \epsilon_{r-n}(r)$. Hence 


$$
\delta_{r}=\sum_{n=1}^{r} C_{r-n} \epsilon_{n}(r)+C_{r}
$$

and the determinental expression for $C_{N}$ is now obtained by applying Cramer's rule to the above system of equations with $0 \leq r \leq N$. This concludes the proof of Theorem 1 .

Let us now show that $\lim _{q \rightarrow 1} \epsilon_{j}(r)(q)_{j}^{-1}=0$ for $0<j \leq r$.

$$
\begin{aligned}
\lim _{q \rightarrow 1} \epsilon_{j}(r)(q)_{j}^{-1} & =- \text { Coefficient of } t^{j-2} \text { in }\left(\begin{array}{c}
r-1 \\
j
\end{array}\right) \cdot\left[\frac{d}{d t}\left(t^{-1} \phi(t)\right)\right] \phi(t)^{j-1} \\
& =- \text { Coefficient of } t^{j-2} \text { in } \frac{1}{j}\left(\begin{array}{c}
r-1 \\
j
\end{array}\right) t^{j-1} \frac{d}{d t}\left(t^{-1} \phi(t)\right)^{j} \\
& =- \text { Coefficient of } t^{-1} \text { in } \frac{1}{j}\left(\begin{array}{c}
r-1 \\
j
\end{array}\right) \frac{d}{d t}\left(t^{-1} \phi(t)\right)^{j}=0
\end{aligned}
$$

since $d t^{r} / d t \neq C t^{-1}$ for any $r$ with $C \neq 0$ and since $\left(t^{-1} \phi(t)\right)^{j}=\Sigma_{r=-j}^{\infty} a_{r} t^{r}$. (2.3) may now be applied to show that

$$
\lim _{q \rightarrow 1} \frac{C_{n}}{(q)_{n}}=\lim _{q \rightarrow 1} \frac{\delta_{n}}{(q)_{n}}=n !^{-1}\left[\frac{d^{n-1}}{d t^{n-1}}\left(f^{\prime}(t)(\phi(t))^{n}\right)\right]_{t=0} .
$$

Since $\epsilon_{r}(r) \equiv 0$ (due to the fact that $\Delta_{(0)} f(t) \equiv 0$ ), we see that $\delta_{1}=c_{1}$ and so (2.4) is clearly true for $n=1$. If we assume (2.4) to be valid up to, but not including a particular $n$, then, by (2.2),

$$
\frac{\delta_{n}}{(q)_{n}}=\sum_{j=1}^{n-1} \frac{C_{n-j}}{\left(q^{j+1}\right)_{n-j}} \frac{\epsilon_{j}(n)}{(q)_{j}}+\frac{C_{n}}{(q)_{n}}
$$

Consequently, by the induction hypothesis,

$$
\begin{aligned}
\lim _{q \rightarrow 1} \frac{C_{n}}{(q)_{n}} & =\lim _{q \rightarrow 1} \frac{\delta_{n}}{(q)_{n}}-\sum_{j=1}^{n-1} \frac{(n-j) ! j !}{n !}\left(\lim _{q \rightarrow 1} \frac{C_{n-j}}{(q)_{n-j}}\right)\left(\lim _{q \rightarrow 1} \frac{\epsilon_{j}(n)}{(q)_{j}}\right) \\
& =\lim _{q \rightarrow 1} \frac{\delta_{n}}{(q)_{n}}-\sum_{j=1}^{n-1} \frac{j !}{n !}\left[\frac{d^{n-j-1}}{d t^{n-j-1}}\left(f^{\prime}(t)(\phi(t))^{n-j}\right)\right]_{t=0} \cdot 0 \\
& =\lim _{q \rightarrow 1} \frac{\delta_{n}}{(q)_{n}}=n !^{-1}\left[\frac{d^{n-1}}{d t^{n-1}}\left(f^{\prime}(t)(\phi(t))^{n}\right)\right]_{t=0} \cdot
\end{aligned}
$$

3. The Carlitz expansion (1.2). We now apply Theorem 1 to the special case $\phi(x)=1-a x, k=1$. In this case, when $j<r$, 


$$
\begin{aligned}
\epsilon_{j}(r) & =\frac{-(q)_{r-1} q^{r-j}}{(q)_{r-j}}\left\{\text { Coefficient of } t^{j-2}\right. \text { in } \\
& \left.t^{-1}\left(t^{-1}(1-a t q)-t^{-1} q^{-r+j}\left(1-a t q^{r-j+1}\right)\right)\left(a t q^{r-j+2}\right)_{j-1}\right\} \\
& =\frac{-(q)_{r-1} q^{r-j}}{(q)_{r-j}}\left\{\text { Coefficient of } t^{j-2} \text { in } t^{-2}\left(1-q^{-r+j}\right)\left(a t q^{r-j+2}\right)_{j-1}\right\} \\
& =0
\end{aligned}
$$

since the highest power of $t$ in $t^{-2}\left(1-q^{-r+j}\right)\left(a t q^{r-j+2}\right)_{j-1}$ is $t^{j-3}$. Hence, by Theorem $1, C_{n}=\delta_{n}$, and we have

$$
F(x)=F(0)+\sum_{n \geq 1} \frac{x^{n}}{(q)_{n}(a x q)_{n}}\left[\Delta^{n-1}\left(\Delta F(t) \cdot\left(\text { atq }_{n}\right)\right]_{t=0} \cdot\right.
$$

When $a=q^{-1}$ we get (1.2).

4. Evaluation of the $C(n \mid q)$. We now treat the case originally envisioned by Carlitz; namely (1.3) where now $F(x)=x, \phi(x)=(1-x)^{-1}$.

Theorem 2. Let $C(n \mid q)$ be defined by (1.2), then

$$
\begin{aligned}
& q^{2 n-2} \frac{(1-q)}{1-q^{n}}\left(\begin{array}{c}
2 n-2 \\
n-1
\end{array}\right)=C(n-1 \mid q) \\
&-q \sum_{j=1}^{n}\left(1-q^{n-j}\right) q^{(n-j+1)(j-1)}\left(\begin{array}{c}
2 j-1 \\
j-1
\end{array}\right)_{q} C(n-1-j \mid q) .
\end{aligned}
$$

Remark. This result exhibits clearly that $C(n-1 \mid 1)=n^{-1}\left(\begin{array}{c}2 n-2 \\ n-1\end{array}\right)$.

Proof. We assert that (4.1) is essentially (2.2) when $\phi(x)=(1-x)^{-1}$, $k=2$. This is because in this case

$$
\delta_{r}=\left[\Delta^{r-1}\left((\Delta t) \cdot\left(t q^{2}\right)_{r}^{-1}\right)\right]_{t=0}=q^{2 r-2}(1-q)\left(1-q^{2 r-2}\right) \ldots\left(1-q^{r}\right),
$$

and

$$
\begin{aligned}
& \epsilon_{j}(r)=-(q)_{r-1} q^{r-j} \\
& \cdot\left\{\text { Coefficient of } t^{j-2} \text { in } \frac{\left[1-(1-t q) / q^{r-j}\left(1-t q^{r-j+1}\right)\right]}{t^{2}\left(t q^{r-j+2}\right)_{j}}\right\}(q)_{r-j}^{-1} \\
&=(q)_{r-1}\left\{\text { Coefficient of } t^{j-2} \text { in } \frac{\left(1-q^{r-j}\right)\left(1-t q-t q^{r-j+1}\right)}{t^{2}\left(t q^{r-j+1}\right)_{j+1}}\right\}(q)_{r-j}^{-1} \\
&=(q)_{r-1}\left\{\text { Coefficient of } t^{j}\right. \text { in } \\
&=(q)_{r-1}\left\{\left(1-q^{r-j}\right)\left(1-t q\left(1+q^{r-j}\right)\right) \sum_{n \geq 0}\left(\begin{array}{c}
j+n \\
n
\end{array}\right)_{q} t^{n} q^{(r-j+1) n}\right\}_{(q)^{-1}} \\
&-q\left(\begin{array}{c}
2 j \\
j
\end{array}\right)_{q} q^{(r-j+1) j} \\
&-q\left(1-q^{2(r-j))}\left(\begin{array}{c}
2 j-1 \\
j-1
\end{array}\right)_{q} q^{(r-j+1)(j-1)}\right\}(q)_{r-j}^{-1} .
\end{aligned}
$$


Hence, dividing (2.2) by $(q)_{r}$ and noting that $C_{n}=C(N-1 \mid q)(q)_{n}$, we obtain

$$
\begin{aligned}
q^{2 r-2} \frac{(1-q)}{1-q^{r}}\left(\begin{array}{c}
2 r-2 \\
r-1
\end{array}\right)_{q} & =C(r-1 \mid q) \\
+ & \sum_{j=1}^{r} \frac{\left(1-q^{r-j}\right)}{1-q^{r}}\left\{\left(\begin{array}{c}
2 j \\
j
\end{array}\right)_{q} q^{(r-j+1) j}-q\left(1+q^{r-j}\right)\left(\begin{array}{c}
2 j-1 \\
j-1
\end{array}\right)_{q} q^{(r-j+1)(j-1)}\right\} C(r-1-j \mid q) \\
& =C(r-1 \mid n)-q \sum_{j=1}^{r}\left(1-q^{r-j}\right) q^{(r-j+1)(j-1)}\left(\begin{array}{c}
2 j-1 \\
j-1
\end{array}\right)_{q} C(r-1-j \mid q),
\end{aligned}
$$

as desired.

We can, of course, obtain a determinental expression for $C(n-1 \mid q)$ as given by Theorem 1; however Theorem 2 seems to most clearly exhibit the relationship between $C(n-1 \mid q)$ and the $q$-Catalan number $\left(\begin{array}{c}n \\ 1\end{array}\right) q_{q}^{-1}\left(\begin{array}{c}2 n-2 \\ n-1\end{array}\right) q$.

\section{REFERENCES}

1. T. J. I'A. Bromwich, An introduction to the theory of infinite series, 2nd ed., MacMillan, London, 1959.

2. L. Carlitz, Sequences, paths, ballot numbers, Fibonacci Quart. 10 (1972), no. 5, 531-549. MR 47 \#6498.

3. - Some q-expansion formulas, Glasnik Mat. 8 (28) (1973), 205-214.

4. - Problem: q-analog of the Lagrange expansion, from Abstracts and Problems from the Conference on Eulerian Series and Applications, May 1974, Pennsylvania State University.

DEPARTMENT OF MATHEMATICS, PENNSYLVANIA STATE UNIVERSITY, UNIVERSITY PARK, PENNSYLVANIA 16802

Current address: Mathematics Research Center, 610 Walnut Street, Madison, Wisconsin 53706 